\title{
Morphological Patterns of Elastic and Reticulum Fibers in Breast Lesions
}

\author{
Abdelbaset Mohamed Elasbali', Ziad Al-0nzi². Alneil Hamza1, Ezeldine Khalafalla1, \\ Hussain Gadelkarim Ahmed ${ }^{3}$ \\ ${ }^{1}$ Department of Clinical Laboratory Sciences, College of Applied Medical Sciences, Jouf University, Qurayyat, KSA \\ ${ }^{2}$ Department of Clinical Laboratory Sciences, College of Applied Medical Sciences, Jouf University, Skaka, KSA \\ ${ }^{3}$ Department of Pathology, College of Medicine, University of Hail, Hail, KSA \\ Email: hussaingad5@gmail.com
}

How to cite this paper: Elasbali, A.M. Al-Onzi, Z., Hamza, A., Khalafalla, E. and Ahmed, H.G. (2018) Morphological Patterns of Elastic and Reticulum Fibers in Breast Lesions. Health, 10, 1625-1633. https://doi.org/10.4236/health.2018.1012122

Received: November 7, 2018

Accepted: November 30, 2018

Published: December 3, 2018

Copyright (C) 2018 by authors and Scientific Research Publishing Inc. This work is licensed under the Creative Commons Attribution International License (CC BY 4.0).

http://creativecommons.org/licenses/by/4.0/

cc) (i) Open Access

\begin{abstract}
Background: The difficulty with histopathology diagnosis is the presence of numerous benign reactive breast lesions with morphological features mimic malignant lesions. Thus, the objective of this study was to assess the merit of morphological patterns of elastic and reticular fibers in differential diagnosis of benign and malignant breast lesions. Methodology: Fifty biopsies were obtained from females with breast Lesions ( 25 breast carcinoma and 25 benign lesions), their ages ranging from 17 to 85 years with mean age of 39 years old. Morphologic demonstrations of elastic and reticular fibers were performed using conventional histochemical procedures. Results: Intense grades of elastic fiber staining were achieved with carcinoma tissues. Dwindled grades of elastic fiber staining were detected with fibrocystic changes. Elastic fibers in breast carcinoma are significantly increased compared to benign breast lesions $\mathrm{P}<0.05$. Reticular fibers have indicated relatively similar presentation in benign and malignant breast lesions. Conclusion: Histochemical quantifications of elastic and reticular fibers can assist in routine diagnosis of breast lesions. Elastic fibers significantly increase in breast carcinoma compared to benign breast lesions.
\end{abstract}

\section{Keywords}

Breast Cancer, Benign Breast Lesions, Reticular Fibers, Elastic Fibers

\section{Introduction}

Breast cancer represents the top cancer-interrelated mortality in women around the globe. About $70 \%$ of breast cancer related deaths subsist in the developing low-income and middle-income countries [1]. Breast cancer is the leading fe- 
males' cancer in the majority of Arab countries and the diseases is increasingly affecting younger women. Still, many patients present with advanced stages of the disease in various Arab countries including Saudi Arabia and Egypt [2]. The increasing rates of breast cancer mortality in developing countries are attributed to late diagnosis of the disease and accessibility obstacles to medical care [3].

Screening and early diagnosis of breast cancer are known to decrease the overall diseases mortality [4]. So the overall breast cancer management can be upgraded through realistic and easy approach interventions. Breast screening for early detection and accurate diagnosis followed by comprehensive treatment can strongly enhance the disease outcomes [4]. Histopathology diagnosis represents the gold standard method for breast cancer diagnosis [5]. The difficulty with histopathology diagnosis is the presence of numerous benign reactive breast lesions with morphological features mimic malignant lesions. Such conditions represent a real diagnostic challenge to even highly experienced histopathologists [6]. In many patients attending with breast lesions, the majority will be diagnosed with benign breast lesions tracked with high risk lesions then emanate malignant lesions [7].

Elastic fibers exist in various organs of the human body, where it offers organs elasticity. These fibers are mainly formed of elastin and elastic fiber microfbrils [8]. Presence of variable quantities of elastic fibers was documented in benign and malignant breast lesions using immunohistochemistry and electron microscopy [9]. Reticular fibers, which are thought to be a form of collagen type III with carbohydrate rich matrix, are consisted of very thin fibers. These fibers form a supporting network for highly cellular tissues [10]. Reticular fibers in capricious quantities were reported in breast tumor-like lesions, as well as, in breast carcinoma [11]. Consequently, quantification of these fibers in benign and malignant lesions by conventional histochemical methods may reduce the complexity of routine differential histopathological diagnosis of benign and malignant breast lesions. Thus, the objective of this study was to assess the merit of morphological patterns of elastic and reticular fibers in differential diagnosis of benign and malignant breast lesions.

\section{Materials and Methods}

In this study 50 formalin fixed paraffin wax embedded tissue blocks (belong to women attended with breast lesions) were retrieved from histopathology laboratory ( 25 were breast cancer and 25 were with benign breast lesions). All biopsies were obtained from females with breast Lesions, their ages ranging from 17 to 85 years with mean age of 39 years old. Patients with breast cancer $(n=25)$ were ascertained as case group, and those with benign breast lesions were ascertained as control group $(\mathrm{n}=25)$. Patients' data were retrieved from laboratory records in Ribat University Hospital, Khartoum state-Sudan.

The previously diagnosed tissue specimens were reconfirmed by pathologist.

Three 3-microns thickness sections were made from each patient's block using 
Rotary Microtome. Out of the 3 sections, one was stained using Hematoxylin and Eosin ( $\mathrm{H} \& \mathrm{E}$ ) (to confirm histopathology diagnosis), one stained using Verhoeff's procedure (for elastic fibers = black color), and the remaining one was stained using Silvers method (Gordon \& Sweet's (for reticular fiber = black color), H \& E (Mayer's technique), Verhoeff's method, and Silver method were applied adopting procedures described elsewhere [12]. Beside the clinicopathological features of the patients, the elastic and reticular fibers were quantitated according to the intensity of the staining into: Negative $(-v e)=$ denotes no staining of fibers; Positive plus one $(+\mathrm{ve})=$ minimum staining; $(++\mathrm{ve})=\bmod -$ erate staining intensity; $(+++v e)=$ deep staining intensity.

\subsection{Ethical Consent}

The study was approved by ethical committee at college of medicine, university of Hail. This in addition to an agreement letter taken from Ribat University Hospital authority.

\subsection{Statistical Analysis}

Data were analyzed using SPSS software version 16 . P value $<0.05$ was considered statistically significant. Frequencies, percentages and cross-tabulations were also produced using the software.

\section{Results}

The present study compared 25 breast cancer tissues obtained from females' patients with breast cancer to 25 benign breast tissues for the presence of elastic and reticular connective tissue fibers. All of the 25 breast cancer cases were diagnosed with ductal carcinoma. The 25 cases of benign breast lesions included, 19 (76\%) fibrocystic changes, 4 (16\%) fibroadenoma, 1 (4\%) papilloma and 1 (4\%) fat necrosis.

About 36\% ( $\mathrm{n}=9)$ of the patients with carcinoma were found at age range 36 - 45 years, $32 \%(n=8)$ at age group $>56$ years, and $16 \%(n=4)$ at age series 25 35 years, as point out in Table 1. Most of cases with fibrocystic changes, fibroadenoma, and fat necrosis were seen at younger age ( $<25$ years) representing 9/19 (47.4\%), $2 / 4$ (50\%), and 1/1 (100\%) (Table 1, Figure 1). These findings indicate

Table 1. Age by types of breast lesion.

\begin{tabular}{ccccccc}
\hline Lesion type & $<25$ years & $\mathbf{2 6 - 3 5}$ & $\mathbf{3 6 - 4 5}$ & $\mathbf{4 6 - 5 5}$ & $>56$ & Total \\
\hline Carcinoma & 1 & 4 & 9 & 3 & 8 & 25 \\
Fibrocystic changes & 9 & 3 & 3 & 3 & 1 & 19 \\
Fibroadenoma & 2 & 2 & 0 & 0 & 0 & 4 \\
Papilloma & 0 & 0 & 0 & 1 & 0 & 1 \\
Fat necrosis & 1 & 0 & 0 & 0 & 0 & 1 \\
Total & 13 & 9 & 12 & 7 & 9 & 50
\end{tabular}




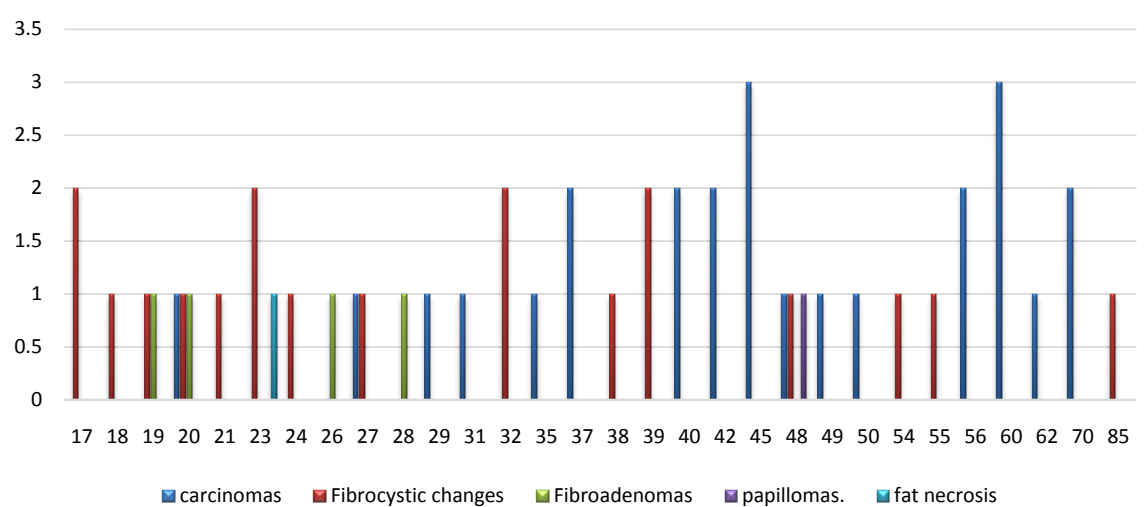

Figure 1. Age by breast lesion type.

that the risk of breast cancer increase with increasing of age and this was found to be statistically significant $\mathrm{P}<0.001$.

Intense grades of elastic fiber staining were achieved with carcinoma tissues. These included $36 \%(\mathrm{n}=9)$ with $(++\mathrm{ve}), 32 \%(\mathrm{n}=8)$ with $(+\mathrm{ve}), 28 \%(\mathrm{n}=7)$ with $(+++v e)$ and only $4 \%(n=1)$ with $(-v e)$. Dwindled grades of elastic fiber staining were detected with fibrocystic changes. These include, $36.8 \%(\mathrm{n}=7)$ with $(-\mathrm{ve}), 31.6 \%(\mathrm{n}=6)$ with $(+\mathrm{ve}), 31.6 \%(\mathrm{n}=6)$ with $(++\mathrm{ve})$ and none with $(+++v e)$. Fibroadenoma acted identical staining intensity in all grades. These outcomes proven the significant intense appearance of elastic fibers in breast carcinoma compared to benign breast lesions and this was found to be statistically significant $\mathrm{P}<0.05$, as indorsed in Table 2, Figure 2, Figure 3 .

Intense grades reticular fiber staining were reached with carcinoma tissues. These included $44 \%(\mathrm{n}=11)$ with $(++\mathrm{ve}), 32 \%(\mathrm{n}=8)$ with $(++\mathrm{ve}), 16 \%(\mathrm{n}=4)$ with $(+v e)$ and only $8 \%(n=2)$ with $(-v e)$. Intense grades of elastic fiber staining were also perceived with fibrocystic changes. These include, $42 \%(\mathrm{n}=8)$ with $(+++v e), 42 \%(\mathrm{n}=8)$ with $(++\mathrm{ve}), 16 \%(\mathrm{n}=3)$ with $(+\mathrm{ve})$ and none with $(-\mathrm{ve})$. Fibroadenoma was significantly detected with intense staining $75 \%(\mathrm{n}=3)$ with $(+++\mathrm{ve})$ and $25 \%(\mathrm{n}=1)$ with $(+\mathrm{ve})$. These results proven the insignificant of intense appearance of elastic fibers in breast carcinoma compared to benign breast lesions and this was found to be statistically insignificant $\mathrm{P}>0.15$, as validated in Table 3, Figure 4, Figure 5.

\section{Discussion}

Extra reproducible diagnostic evidences for differential histopathological diagnosis of breast lesions will minimize the challenges facing histopathologists to differentiate between dubious breast lesions. In order to boost diagnostic evidence using conventional histopathology, which still used as a routine for initial diagnosis of tissue biopsy, the current study test the merit of elastic and reticular fibers in differential diagnosis of breast lesions.

The outcomes of the present study proven the significant intense manifestation of elastic fibers in breast carcinoma compared to benign breast lesions $\mathrm{P}<$ 0.05 . The presence of abundance elastic tissue in breast carcinoma was previously 
Table 2. Breast lesion type by intensity of elastic fibers staining.

\begin{tabular}{cccccc}
\hline Lesion type & Negative (-ve) & Positive (+ve) & ++ve & +++ve & Total \\
\hline Carcinoma & 1 & 8 & 9 & 7 & 25 \\
Fibrocystic changes & 7 & 6 & 6 & 0 & 19 \\
Fibroadenoma & 1 & 1 & 1 & 1 & 4 \\
Papilloma & 1 & 0 & 0 & 0 & 1 \\
Fat necrosis & 0 & 1 & 0 & 0 & 1 \\
Total & 10 & 16 & 16 & 8 & 50 \\
\hline
\end{tabular}

Table 3. Breast lesion type by intensity of reticular fibers staining.

\begin{tabular}{cccccc}
\hline Lesion type & Negative (-ve) & Positive (+ve) & ++ve & +++ve & Total \\
\hline Carcinoma & 2 & 4 & 11 & 8 & 25 \\
Fibrocystic changes & 0 & 3 & 8 & 8 & 19 \\
Fibroadenoma & 0 & 1 & 0 & 3 & 4 \\
Papilloma & 0 & 0 & 1 & 0 & 1 \\
Fat necrosis & 0 & 0 & 0 & 1 & 1 \\
Total & 2 & 8 & 20 & 20 & 50
\end{tabular}

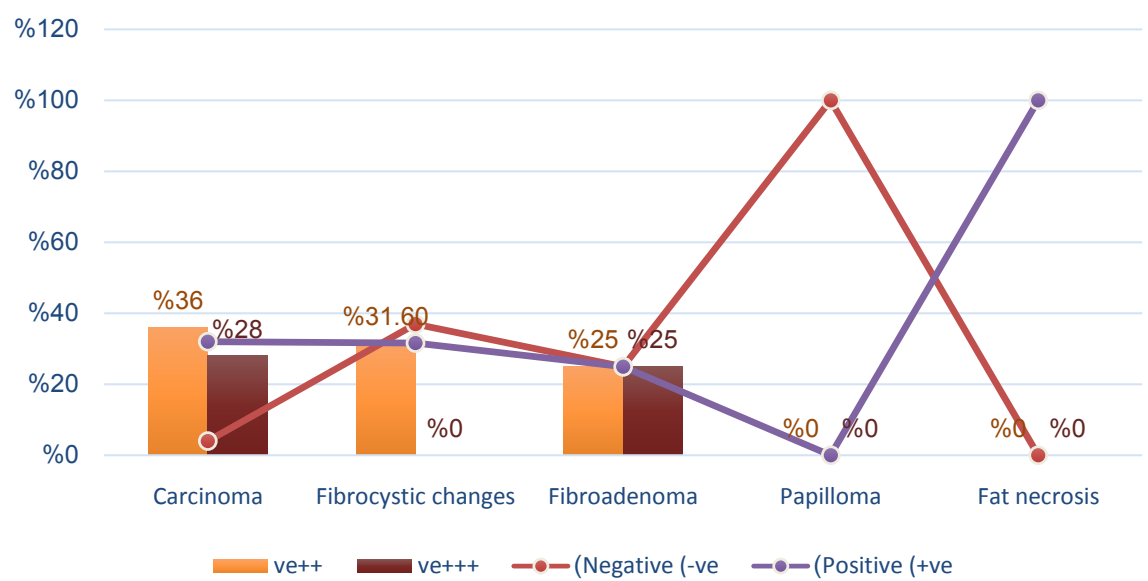

Figure 2. Breast lesion type by intensity of elastic fibers staining.

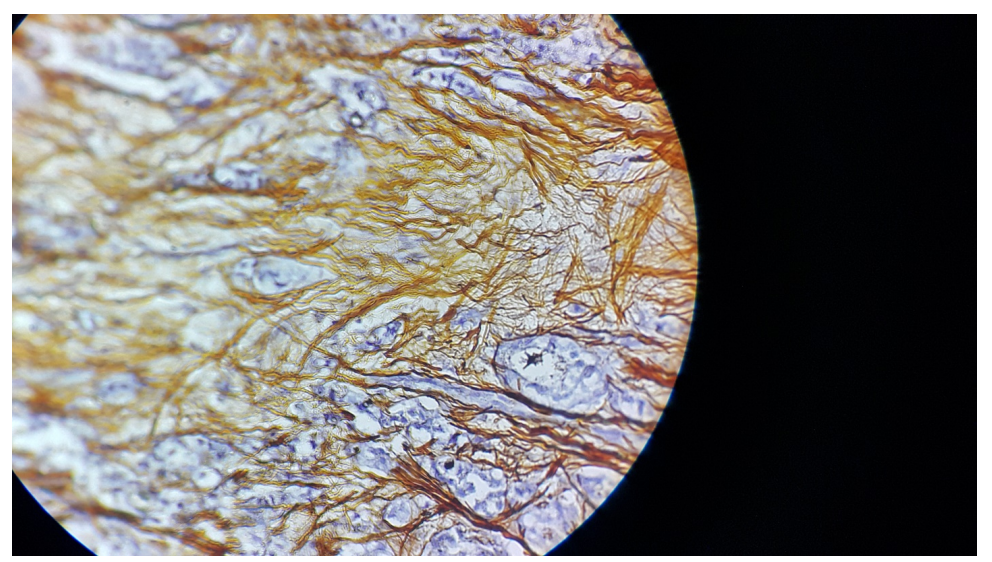

Figure 3. Breast cancer showing elastic fiber (+ve) stained in Verhoeff's procedure. 


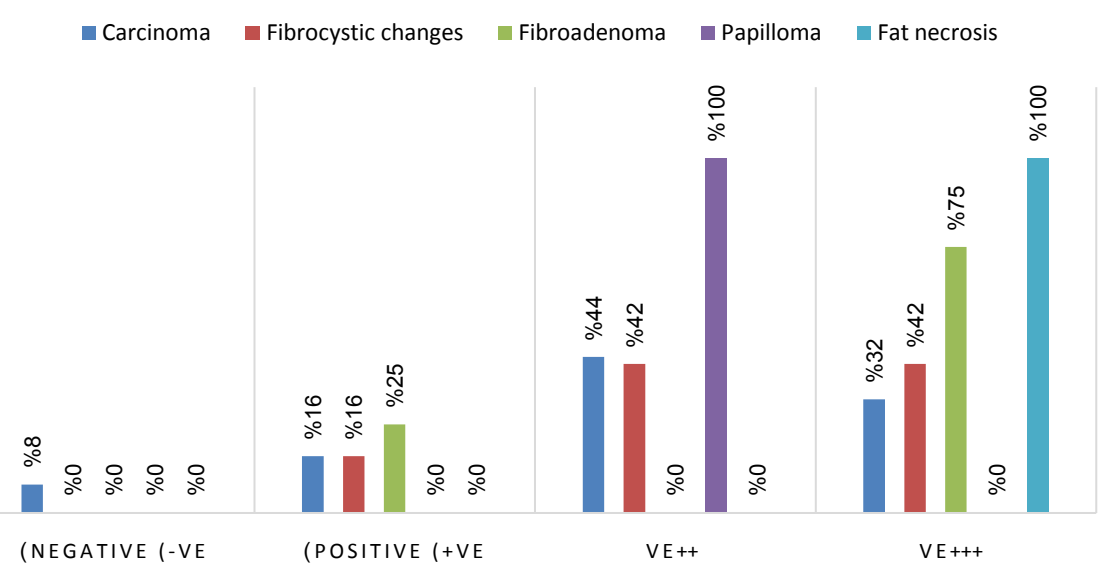

Figure 4. Breast lesion type by intensity of reticular fibers staining.

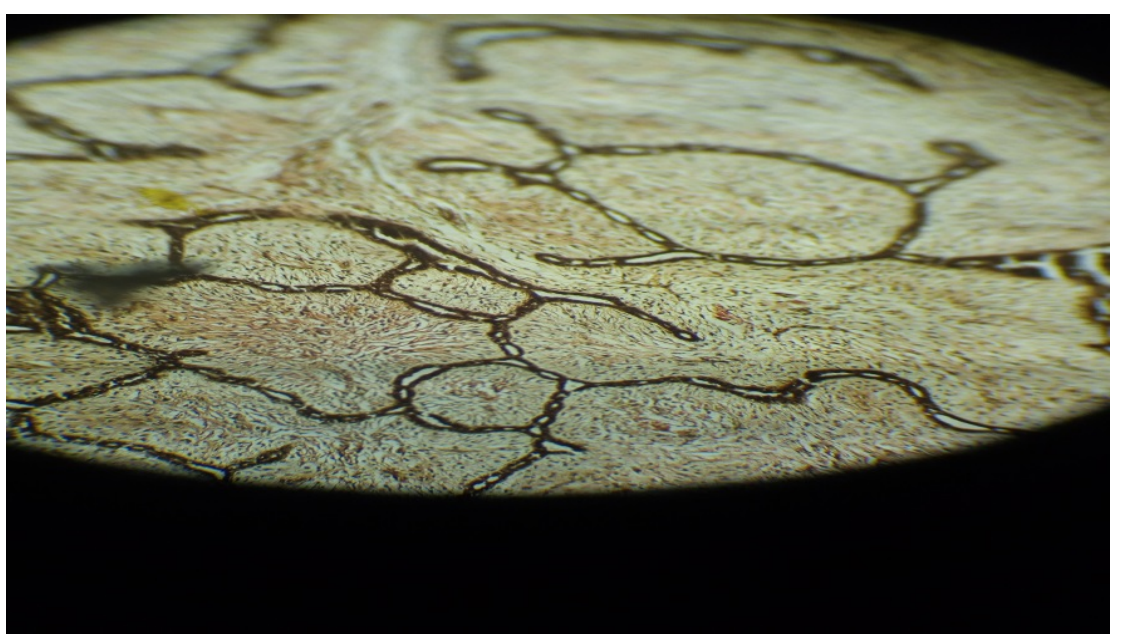

Figure 5. Breast cancer showing reticular fibers (++ve) stained in Silvers method (Gordon \& Sweet's).

reported. Though elastic may exist in benign breast lesions, elastic fibers tissue is predominantly periductal in ductal carcinoma [12]. It was suggested that the presence of elastosis is an important feature in differentiation between histoprognostic grades 1 and 2 of low degree breast cancer [13]. Elastosis was also described in about $90 \%$ of infiltrating ductal and lobular breast cancers, this in addition to its present in benign breast lesions, as well as, non-breast tumors [14] [15]. However, recent studies using advance technology such as CHear-Wave Elastography (SWE), facilitated the differentiation between benign and malignant breast lesions using elastic fibers morphological characteristics. Quantitative assessment of the elastic tissue displays discriminatory capability in differentiating Ultrasound diagnosed breast lesions and could be stratify low suspicion lesions within Breast Imaging Reporting and Data System to evade superfluous biopsy [16].

In this study, some of the cases with fibrocystic changes and fibroadenoma have expressed equitable quantities of elastic tissues. Fibrocystic breast disease is the most breast cellular proliferative disease [17]. The fibrocystic disease is asso- 
ciated with hyperproliferation of connective tissue due to high proportion of estrogen and deficiency of progesterone, which was found to increase the risk of breast cancer 2 - 4 folds [18] [19]. On the other hand, a previous study has reported the presence of elastic fibers in fibroadenoma, but when compared to breast carcinoma, breast carcinoma explicit more fibrous density [20].

Reticular fibers have indicated relatively similar presentation in benign and malignant breast lesions. As these fibers are unremitting with collagen fibers [21], though reticular fibers have unlike histochemical reactions [22]. It was well established that breast cancer is associated with increased accumulation of reticular and collagen fibers [23]. Breast malignant cells were found to recruit the stromal cells fibroblasts (forming fibronectin and collagen I) to amend their extracellular matrix and endorse tumor growth and subsequent invasion [24]. Fibrocystic changes as well as, fibroadenoma are known to harbor accumulation of connective tissues including collagen and its related reticular fiber [25] [26]. However, there is a lack of literature regarding the characterization of reticular fibers, which necessitate more work in this track.

\section{Conclusion}

Histochemical quantifications of elastic and reticular fibers can assist in routine diagnosis of breast lesions. Elastic fibers significantly increase in breast carcinoma compared to benign breast lesions. Further research is required in respect to cost-effective histological technology, which might assist where un-affordable to sophisticated diagnostic methods exist.

\section{Conflicts of Interest}

The authors declare no conflicts of interest regarding the publication of this paper.

\section{References}

[1] Lukong, K.E., Ogunbolude, Y. and Kamdem, J.P. (2017) Breast Cancer in Africa: Prevalence, Treatment Options, Herbal Medicines, and Socioeconomic Determinants. Breast Cancer Research and Treatment, 166, 351-365.

https://doi.org/10.1007/s10549-017-4408-0

[2] Unger-Saldaña, K. (2014) Challenges to the Early Diagnosis and Treatment of Breast Cancer in Developing Countries. World Journal of Clinical Oncology, 5, 465-477. https://doi.org/10.5306/wjco.v5.i3.465

[3] Anderson, B.O. and Jakesz, R. (2008) Breast Cancer Issues in Developing Countries: An Overview of the Breast Health Global Initiative. Breast cancer Issues in Developing Countries: An Overview of the Breast Health Global Initiative. World Journal of Surgery, 32, 2578-2585. https://doi.org/10.1007/s00268-007-9454-z

[4] Cedolini, C., Bertozzi, S., Londero, A.P., et al. (2014) Type of Breast Cancer Diagnosis, Screening, and Survival. Clinical Breast Cancer, 14, 235-240. https://doi.org/10.1016/j.clbc.2014.02.004

[5] Saco, A., Ramírez, J., Rakislova, N., Mira, A. and Ordi, J. (2016) Validation of Whole-Slide Imaging for Histolopathogical Diagnosis: Current State. Pathobiology, 
83, 89-98. https://doi.org/10.1159/000442823

[6] Torous, V.F., Schnitt, S.J. and Collins, L.C. (2017) Benign Breast Lesions That Mimic Malignancy. Pathology, 49, 181-196.

https://doi.org/10.1016/j.pathol.2016.12.002

[7] Moon, H.J., Kim, M.J., Yoon, J.H. and Kim, E.K. (2016) Risks of Being Malignant or High Risk and Their Characteristics in Breast Lesions $20 \mathrm{~mm}$ or Larger after Benign Results on Ultrasonography-Guided 14-Gauge Core Needle Biopsy. Ultrasound Q, 32, 157-163. https://doi.org/10.1097/RUQ.0000000000000179

[8] Uitto, J. (1979) Biochemistry of the Elastic Fibers in Normal Connective Tissues and its Alterations in Diseases. Journal of Investigative Dermatology, 72, 1-10. https://doi.org/10.1111/1523-1747.ep12530093

[9] Uchiyama, S. and Fukuda, Y. (1989) Abnormal Elastic Fibers in Elastosis of Breast Carcinoma. Ultrastructural and Immunohistochemical Studies. Acta Pathological Japonica, 39, 245-253.

[10] Textor, J., Mandl, J.N. and de Boer, R.J. (2016) The Reticular Cell Network: A Robust Backbone for Immune Responses. PLoS Biology, 14, e2000827. https://doi.org/10.1371/journal.pbio.2000827

[11] Bancroft, J.D. and Gamble, M. (2011) Theory and Practice of Histological Techniques. 6th Edition, CHurchil Livingstone/Elsevier, London, 121-135.

[12] Tremblay, G., Buell, R.H. and Seemayer, T.A. (1977) Elastosis in Benign Sclerosing Ductal Proliferation of the Female Breast. The American Journal of Surgical Pathology, 1, 155-166. https://doi.org/10.1097/00000478-197706000-00006

[13] Rolland, P.H., Jacquemier, J. and Martin, P.M. (1980) Histological Differentiation in Human Breast Cancer Is Related to Steroid Receptors and Stromal Elastosis. Cancer Chemotherapy and Pharmacology, 5, 73-77. https://doi.org/10.1007/BF00435407

[14] Isaacson, C., Greeff, H., Murray, J.F., Posen, J. and Schmaman, A. (1985) Elastosis in Malignant Tumours. South African Medical Journal, 68, 30-32.

[15] Raju, G.C. and Lee, Y.S. (1988) Elastosis in the Male Breast. Histopathology, 12, 203-209. https://doi.org/10.1111/j.1365-2559.1988.tb01930.x

[16] Xiao, Y., Yu, Y., Niu, L., et al. (2016) Quantitative Evaluation of Peripheral Tissue Elasticity for Ultrasound-Detected Breast Lesions. Clinical Radiology, 71, 896-904. https://doi.org/10.1016/j.crad.2016.06.104

[17] Talaei, A., Moradi, A. and Rafiei, F. (2017) The Evaluation of the Effect of Metformin on Breast Fibrocystic Disease. Breast Disease, 37, 49-53. https://doi.org/10.3233/BD-160256

[18] Vorherr, H. (1986) Fibrocystic Breast Disease: Pathophysiology, Pathomorphology, Clinical Picture, and Management. American Journal of Obstetrics \& Gynecology, 154, 161-179. https://doi.org/10.1016/0002-9378(86)90421-7

[19] De León Antoni, E. (1989) Is Fibrocystic Disease Precancerous? Boletín de la Asociación Médica de Puerto Rico, 81, 451-454.

[20] Zakout, Y.M., Abdullah, S.M. and Ali, M.A. (2012) Assessment of Elastosis in Invasive Ductal Carcinoma of the Breast Compared to Fibroadenoma among Sudanese Patients Using Conventional Histochemical Methods. Biotechnic \& Histochemistry, 87, 122-125.

[21] Ushiki, T. (2002) Collagen Fibers, Reticular Fibers and Elastic Fibers. A Comprehensive Understanding from a Morphological Viewpoint. Archives of Histology and Cytology, 65, 109-126. https://doi.org/10.1679/aohc.65.109

[22] Snodgrass, M.J. (1977) Ultrastructural Distinction between Reticular and Collagen- 
ous Fibers with an Ammoniacal Silver Stain. The Anatomical Record, 187, 191-205. https://doi.org/10.1002/ar.1091870206

[23] Hamad, A.M. and Ahmed, H.G. (2016) Association of Connective Tissue Fibers with Estrogen Expression in Breast Lesions among Sudanese Females. International Clinical Pathology Journal, 2, 97-102.

[24] Wang, K., Wu, F., Seo, B.R., et al. (2016) Breast Cancer Cells Alter the Dynamics of Stromal Fibronectin-Collagen Interactions. Matrix Biology, 60-61, 86-95. https://doi.org/10.1016/j.matbio.2016.08.001

[25] Anastassiades, O.T., Tsakraklides, E. and Gogas, J. (1981) The Histology of Fibrocystic Disease of the Female Breast. Correlation with Epithelial Proliferative Lesions and Carcinoma in Situ. Pathology-Research and Practice, 172, 109-129. https://doi.org/10.1016/S0344-0338(81)80127-6

[26] Sudheer, G. (2009) Ultrasound Characterization of Breast Masses. The Indian Journal of Radiology and Imaging, 19, 242-247.

https://doi.org/10.4103/0971-3026.54878 